\title{
Potential Role of MRI Imaging for Myofascial Pain: A Scoping Review for the Clinicians and Theoretical Considerations
}

\author{
Valerie Evans ${ }^{1,2}$ \\ Michael Behr 2,3 \\ Anshika Gangwar ${ }^{2}$ \\ Michael D Noseworthy (iD ${ }^{4}$ \\ Dinesh Kumbhare (iD ${ }^{1-3}$ \\ 'Institute of Biomaterials and Biomedical \\ Engineering (IBBME), University of \\ Toronto, Toronto, Ontario, Canada; \\ ${ }^{2}$ University Health Network - Toronto \\ Rehabilitation Institute, Toronto, \\ Ontario, Canada; ${ }^{3}$ Department of \\ Medicine, Division of Physical Medicine \\ and Rehabilitation, University of Toronto, \\ Toronto, Ontario, Canada; ${ }^{4}$ Department \\ of Electrical and Computer Engineering, \\ McMaster University, Hamilton, Ontario, \\ Canada
}

\begin{abstract}
The most common cause of chronic musculoskeletal pain is chronic myofascial pain syndrome (MPS). MPS often presents with increased muscle stiffness, and the myofascial trigger point (MTrP). Imaging modalities have been used to identify the MTrP, but their role in the detection and diagnosis of MPS remains unclear. The purpose of this review was to identify evidence in literature for the use of imaging in the role of classifying and explaining the physiology of MTrPs. Since few imaging techniques have been performed on MTrPs, we explored the imaging techniques that can effectively image complex skeletal muscle microstructure, and how they could be used. As part of a scoping review, we conducted a systematic search from three medical databases (CINAHL, EMBASE and MEDLINE) from year to year to analyze past MTrP imaging, as well as analyzing imaging techniques performed on the microstructure of muscle. Previously, ultrasound has been used to differentiate active, latent MTrPs, but these studies do not adequately address their underlying anatomical structure. MRI remains the standard method of imaging skeletal muscle. The existing MRI literature suggests that the DTI technique can quantify muscle injury, strain, and structure. However, theoretically, HARDI and DKI techniques seem to provide more information for complex structural areas, although these modalities have a disadvantage of longer scan times and have not been widely used on skeletal muscle. Our review suggests that DTI is the most effective imaging modality that has been used to define the microstructure of muscle and hence, could be optimal to image the MTrP. HARDI and DKI are techniques with theoretical potential for analysis of muscle, which may provide more detailed information representative of finer muscle structural features. Future research utilizing MRI techniques to image muscle are necessary to provide a more robust means of imaging skeletal muscle and the MTrP.
\end{abstract}

Keywords: myofascial pain syndrome, MRI, trigger points, chronic pain, imaging

\section{Introduction}

Chronic pain is a critical public health problem, with a prevalence of affecting $11-24 \%$ of the world's population. ${ }^{1}$ Myofascial pain syndrome (MPS) is one of the most common forms of chronic musculoskeletal pain. It is characterized by myofascial trigger points (MTrP), stiffness, reduced range of motion, and altered functional abilities. It has been hypothesized that an MTrP may consist of shortened or angulated muscle fibers with interrupted continuity, often with the center being a fluid-filled region. ${ }^{2}$ MTrPs are defined as hard, discrete, nodules that are located within skeletal muscle taut bands and are usually detected by palpation. They are
Correspondence: Dinesh Kumbhare Department of Medicine and IBBME, University of Toronto, 550 University Ave, Suite 7-|3I, Toronto, ON, M5G 2A2, Canada

Tel + I 4I6-597-3422 × 46I2

Email dinesh.kumbhare@uhn.ca 
known to produce spontaneous pain and can be painful upon palpation. ${ }^{3}$ The etiology of the MTrP remains elusive. But it is probably multifactorial, arising as a result of muscle overuse, unaccustomed exercise, repetitive eccentric exercise, muscle trauma, and/or psychological stress. ${ }^{4}$

According to Simon's integrated hypothesis, the taut band appearance is secondary to the excessive release of acetylcholine in some dysfunctional motor endplates resulting in the contracture. ${ }^{5,6}$ The majority of cases of MPS are found in the neck, parascapular region and upper back muscles in patients. ${ }^{7}$

Diagnosing myofascial pain has been an ongoing challenge in modern day clinical medicine. Rivers et $\mathrm{al}^{8}$ conducted an international survey of International Association for the Study of Pain (IASP) members to establish preliminary clinical diagnostic criteria. Their proposed criteria include decreased range of motion of the affected joints, pain with palpation of the tender area, and worsening symptoms with palpation of a trigger point. This diagnostic criteria provide a list of items that do not have any specific quantitative measurements or any clear relationship to underlying pathophysiology. We believe that there is a need to develop/include quantitative biomarkers to improve the overall clinical diagnostic criteria. Ideally, these diagnostic biomarkers would be objective, reliable, reproducible and reflect underlying pathophysiology and/ or disease mechanisms. Examples of quantitative assessments could include measurements of the structural aspects of skeletal muscle such as contracted sarcomere, muscle fiber path deviation, muscle fiber discontinuity, and intramuscular inflammatory change. Presently, however, these measures are not only difficult to acquire but are also not available for use within the clinical setting. If they could be added to the current diagnostic criteria, a pathophysiological perspective to the overall diagnosis would be added which is currently absent. Once developed, they may assist in the construction of more homogenous groups, which could reflect clinical phenotypes, and improve future research methodology and clinical outcomes.

One way to non-invasively acquire quantitative structural measurements of skeletal muscle is by using medical imaging. The imaging modalities that have been previously used on trigger points include ultrasound (US), magnetic resonance elastography (MRE) and infrared thermography. Ultrasound has been used as the primary imaging modality for researching and diagnosing trigger points, a review on this topic has been previously published. ${ }^{9}$ Many studies have found that it is possible to differentiate active, latent MTrPs, and normal tissue by using US imaging. ${ }^{2,5}$ While US imaging provides new and exciting possibilities for identifying physical characteristics of MTrPs on human subjects in vivo, noninvasively and at low cost, ${ }^{10}$ however, it also has drawbacks. Limitations include limited field of view, limited resolution and being heavily, operator dependent. ${ }^{10}$ Furthermore, the evaluation of deep structures may be difficult and have reduced sensitivity in regard to morphologic injury. ${ }^{11}$ Additionally, compared to magnetic resonance imaging (MRI), there is scarcity of data regarding the clinical relevance of US findings in acute muscle injuries. ${ }^{11}$ Infrared thermography has also been explored on muscle trigger points. Cojocaru et $\mathrm{al}^{12}$ established that trigger points have higher temperatures because a contracted muscle band generates heat, leaving a cooler area in its surrounding due to deficits in blood flow. However, these imaging modalities are not ideal to assess the microstructural attributes of the MTrP due to their poor spatial resolution and lack of tomographic representation. Most recently, Gerwin et al $^{13}$ analyzed muscle biopsy samples through light and electron microscopy to identify foci of segmentally contracted sarcomeres in human skeletal muscles and to determine whether these could be associated with trigger points in muscle biopsies taken from myalgic and non-myalgic muscle. Their results revealed the presence of segmentally contracted sarcomeres in human skeletal muscles. However, authors concluded that their findings should be interpreted with caution as they are unable to discern precisely whether the images were a result of actuality or due to artifact. Thereby, showcasing the limitation associated with using this imaging modality. Moreover, Moo and Herzog ${ }^{14}$ used non-linear microscopy to measure sarcomeres at different locations in an intact whole muscle in mice. However, they indicated that images of deep muscle tissues are of poor quality.

Magnetic resonance imaging (MRI) is considered the reference standard imaging method to assess the morphology of muscles due to its capability to visualize soft tissues with excellent contrast, high resolution and multiplanar assessment of muscles, especially in cases where traumatic lesions are clinically suspected. ${ }^{11}$ To date, the only magnetic resonance imaging technique that has been used to directly investigate trigger points is magnetic resonance elastography (MRE). MRE has the potential to improve detection of skeletal muscle stiffness, as it allows 
researchers to assess the biomechanical properties of skeletal muscles. ${ }^{15}$ It has been successfully used to examine both healthy and diseased muscles, as it allows for the calculation of shear moduli and detection of myofascial taut bands.

\section{Knowledge Gap}

The precise anatomical and ultrastructural content of the MTrP has not yet been elucidated. Understanding this would advance our knowledge of the pathophysiology of MPS. Identification of an imaging modality would allow measurements of these aspects thereby advancing researchers' ability to investigate the pathophysiology of the MTrP and MPS and validate or refute the prevailing theories. The integrated hypothesis suggests that a disruption of the muscle causes a neurochemical cascade that forms trigger points ${ }^{16}$ whereas the neurogenic hypothesis suggests that functional mechanisms create neurogenic inflammatory perturbations that cause trigger points. ${ }^{17}$ The integrated hypothesis would suggest that muscle fiber bundle perturbations present near the area of most mechanical stress, particularly at the muscle-tendon junction. Theoretically, these could be the muscle fiber bundles whose sarcomeres are contracted and whose path deviates in the region of the MTrP. The fibers could also be disrupted in that region. The neurogenic hypothesis would result in a pattern of localized muscle fiber contractions conforming to the innervation pattern of the muscle. Thus theoretically, the images produced by these two hypotheses would be different from one another with regard to location and characteristics of abnormalities. The current imaging literature does not provide information about these phenomena. Thus, in order to gain more understanding of these two hypotheses, there is a need to discover the optimal modality to image these MTrPs.

\section{Objectives}

With numerous efforts to quantify the nature and presence of MTrP, multiple modalities have been investigated to best study their diagnostic efficiency, including T2 mapping, diffusion tensor imaging (DTI), diffusion kurtosis imaging (DKI) and high angular resolution diffusion imaging (HARDI). See Appendix A for definitions on each imaging modality. Based on the literature, many of the techniques available focus on identifying the secondary effects of the trigger point such as the stiffness, thermal pattern and surrounding blood supply. Therefore, we set out to investigate whether any imaging modalities have been used to describe the anatomical attributes of the MTrP itself.

\section{Methods}

\section{Literature Search: Finding the Optimal Technique to Image Anatomical and Ultrastructural Attributes of Skeletal Muscle and the Trigger Point}

This literature search was conducted using the library database, using in CINHAL (1982 -2019), EMBASE (1947-2019), and MEDLINE (1965-2019) databases. We began the literature search of articles focusing on MRI techniques to image muscle fibers, trigger points, tractography and muscle microstructure. Prior research on imaging techniques performed on muscle injury and microstructure can infer which modality is most effective when imaging trigger points.

\section{Inclusion Criteria}

Inclusion criteria for this search encompasses T2 mapping, DTI, HARDI and DKI techniques. To be included in this study were peer-reviewed primary studies or review articles that addressed imaging healthy or physically damaged skeletal muscle microstructure from human or animal models of myofascial pain. Also included in the search criteria are comparisons between the different imaging modalities.

\section{Exclusion Criteria}

Exclusion criteria for the search eliminated studies that imaged the following: Effects of surgery on muscle, cardiac muscle, muscle dystrophy, ligament injury, areas of the nervous system, imaging grey or white matter, ligament imaging. Furthermore, we excluded studies if they referred to skeletal muscle that was not easily palpable and not commonly affected by MTrPs. Please refer to the PRISMA diagram in Figure 1 for the systematic review of literature that was performed. A detailed search strategy can be found in Appendix B.

\section{Search Strategy}

Since the structure of a trigger point is extremely complex, finding the modality that can successfully and most accurately image skeletal muscle fibers and microstructure is essential.

Using the OVID database, we began our literature search of articles related to imaging techniques used on 


\section{PRISMA 2009 Checklist}

\begin{tabular}{|c|c|c|c|}
\hline Section/topic & \# & Checklist item & $\begin{array}{l}\text { Reported } \\
\text { on page \# }\end{array}$ \\
\hline \multicolumn{4}{|l|}{ TITLE } \\
\hline Title & 1 & Identify the report as a systematic review, meta-analysis, or both. & 1 \\
\hline \multicolumn{4}{|l|}{ ABSTRACT } \\
\hline Structured summary & 2 & $\begin{array}{l}\text { Provide a structured summary including, as applicable: background; objectives; data sources; study eligibility criteria, } \\
\text { participants, and interventions; study appraisal and synthesis methods; results; limitations; conclusions and } \\
\text { implications of key findings; systematic review registration number. }\end{array}$ & 2 \\
\hline \multicolumn{4}{|l|}{ INTRODUCTION } \\
\hline Rationale & 3 & Describe the rationale for the review in the context of what is already known. & $3-5$ \\
\hline Objectives & 4 & $\begin{array}{l}\text { Provide an explicit statement of questions being addressed with reference to participants, interventions, comparisons, } \\
\text { outcomes, and study design (PICOS). }\end{array}$ & 5 \\
\hline \multicolumn{4}{|l|}{ METHODS } \\
\hline Protocol and registration & 5 & $\begin{array}{l}\text { Indicate if a review protocol exists, if and where it can be accessed (e.g., Web address), and, if available, provide } \\
\text { registration information including registration number. }\end{array}$ & $\begin{array}{l}\text { This is a } \\
\text { scoping } \\
\text { review. }\end{array}$ \\
\hline Eligibility criteria & 6 & $\begin{array}{l}\text { Specify study characteristics (e.g., PICOS, length of follow-up) and report characteristics (e.g., years considered, } \\
\text { language, publication status) used as criteria for eligibility, giving rationale. }\end{array}$ & 6 \\
\hline Information sources & 7 & $\begin{array}{l}\text { Describe all information sources (e.g., databases with dates of coverage, contact with study authors to identify } \\
\text { additional studies) in the search and date last searched. }\end{array}$ & $5-6$ \\
\hline Search & 8 & $\begin{array}{l}\text { Present full electronic search strategy for at least one database, including any limits used, such that it could be } \\
\text { repeated. }\end{array}$ & $\begin{array}{l}\text { Appendix } \\
\text { B }\end{array}$ \\
\hline Study selection & 9 & $\begin{array}{l}\text { State the process for selecting studies (i.e., screening, eligibility, included in systematic review, and, if applicable, } \\
\text { included in the meta-analysis). }\end{array}$ & 7 \\
\hline Data collection process & 10 & $\begin{array}{l}\text { Describe method of data extraction from reports (e.g., piloted forms, independently, in duplicate) and any processes } \\
\text { for obtaining and confirming data from investigators. }\end{array}$ & 7 \\
\hline Data items & 11 & $\begin{array}{l}\text { List and define all variables for which data were sought (e.g., PICOS, funding sources) and any assumptions and } \\
\text { simplifications made. }\end{array}$ & 7 \\
\hline $\begin{array}{l}\text { Risk of bias in individual } \\
\text { studies }\end{array}$ & 12 & $\begin{array}{l}\text { Describe methods used for assessing risk of bias of individual studies (including specification of whether this was } \\
\text { done at the study or outcome level), and how this information is to be used in any data synthesis. }\end{array}$ & $\begin{array}{l}\text { Appendix } \\
\text { C }\end{array}$ \\
\hline Summary measures & 13 & State the principal summary measures (e.g., risk ratio, difference in means). & 7 \\
\hline
\end{tabular}

Figure I PRISMA diagram.

Notes: PRISMA figure adapted from Liberati A, Altman D, Tetzlaff J, et al. The PRISMA statement for reporting systematic reviews and meta-analyses of studies that evaluate health care interventions: explanation and elaboration. Journal of Clinical Epidemiology. 2009;62(10). Creative Commons. ${ }^{60}$

skeletal muscle and tractography. This was accomplished by grouping keywords of imaging modalities and pairing it with skeletal muscle and tractography keywords.

We also included keywords such as "microstructure" and "fiber or fibre" in order to narrow the search to our intended question. These results assisted in determining the best modality to image skeletal fibers and microstructure. After removing duplicates between the three databases, 351 papers were reported for screening. The keywords used for the systematic search are presented in Appendix B.

\section{Data Extraction}

We followed the Cochrane methodology and applied our inclusion and exclusion criteria. ${ }^{18}$ Once the studies were successively extracted, they were independently assessed by two reviewers (VE and AG). Any conflicts were resolved through discussion. The search results are outlined in Figure 1.

\section{Qualitative Assessment of Studies}

From the 40 studies extracted, 28 of them were primary studies, while 12 were types of review articles. The primary studies from the data extraction were reviewed by the Downs and Black ${ }^{19}$ checklist for the assessment of methodological quality. Three of the studies were not evaluated by this checklist as we could not obtain access to those articles. The score is based on 28 questions with scores of poor $(<14)$, fair (15-19), good (20-25), and excellent (25-28). From the assessment, only 12 out of 25 primary articles were evaluated as having "fair" quality. However, many of the questions in the checklist related to the quality of participants recruited, and if the studies included blinding of the participants. Since the studies 
extracted involve the imaging of skeletal microstructure, the blinding of participants is of little importance to the outcome of the study. For these reasons, these studies were rated lower on the checklist. There were also many studies that used animal subjects or very few human participants, because of the difficultly to acquire large human populations for moderately long scan times. The full quality assessment is found in Appendix C.

\section{Results}

Of the 351 filtered in the initial stage, 218 studies were removed after applying our inclusion and exclusion criteria. Of the 133 studies were reviewed in full text, 92 were removed for being irrelevant to our study objectives as they were either using incorrect interventions or incorrect settings. Fourteen of the articles focused on imaging muscles that did not fit our study inclusion and exclusion criteria because of the anatomical location of muscles examined including mastetory, mandibular, pterygoid, pubovisceralis, pelvic muscles and the tongue muscle. Sixteen articles focused on new techniques to optimize the parameters and extract features from noisy environments. Twelve were excluded as they explored characteristics such as muscle associated with a disease or condition including ischemia, diabetes, spine disease, and muscular dystrophy or atrophy. Nine were excluded as they were characterizing fiber type, recruitment or strength benefits from exercise. Six were excluded that focused on imaging muscle metabolites and myosin chain activity. Other studies excluded dealt with tendon injuries, denervated muscles, and age and sex differences within muscles as well as contraction and angle changes within muscle.

\section{T2 Mapping}

Conventionally, clinical studies are done using T2 weighted MR images to quantify muscle characteristics and injuries. More recently, a more robust and quantitative approach has been accepted in the clinic - that of parametric T2 mapping. A multi-echo spin technique is usually applied to measure $\mathrm{T} 2$ values, accurately demonstrating the $\mathrm{T} 2$ increase in relaxation time of water within skeletal muscles. ${ }^{10}$ This technique has the potential to provide useful data about the capacity of a muscle group to be activated, which could be useful in demonstrating early fatty atrophy. ${ }^{20} \mathrm{~T} 2$ mapping also has the potential to image damaged muscle resulting from eccentric exercise. T2 relaxation times can detect differences in muscles for up to 80 days after the injury and can reflect muscle edema as well as the changes in the characteristics of cell water. ${ }^{21}$

Many studies quantify muscle injury using T2 weighted imaging. Quantitative assessment can only be done, however, using T2 mapping. Quantitative studies using a rabbit model have demonstrated that T2 mapping, performed on the affected muscles after trauma, is an effective measure of muscular injury. ${ }^{22,23}$ These findings were also consistent when evaluating compression induced deep tissue injury in rat muscle, ${ }^{24}$ as well as estimating post-operative back muscle injury in rats. ${ }^{25}$

A relationship between muscle soreness and $\mathrm{T} 2$ relaxation has also been investigated. The change that occurs in the T2 decay has been shown to be similar to the changes in the cross-sectional area of that muscle, both reaching a peak value around 12-24 hours post-exercise. ${ }^{26}$ Marqueste et $\mathrm{al}^{27}$ also evaluated enzymatic activity and $\mathrm{T} 2$ relaxation times in various muscles and concluded that both can provide qualitative and quantitative information of muscle damage related to single or repeated bouts of downhill running conducted until exhaustion. T2 values are thought to be an effective measure of muscle soreness because of the edema within the muscle tissue - which is known to significantly increase $\mathrm{T} 2$ relaxation time.

\section{Diffusion Tensor Imaging (DTI) Imaging}

Diffusion tensor imaging has accurately shown muscle structure and injury. ${ }^{26}$ DTI is potentially a powerful tool for the non-invasive study of muscle architecture and fiber tracking. ${ }^{28}$ The application of DTI has most frequently been done in the central nervous system, so a great deal of the methodology for muscle fiber tracking has been borrowed and applied using tools already available. ${ }^{29}$ The DTI approach is able to differentiate between functionally different muscles in the same body region as well as differentiate between injured and uninjured muscle, based on their water diffusive properties. ${ }^{30-32}$

Using fiber tracking, a method that aligns directionality from the primary eigenvectors for each voxel, DTI has also been shown to differentiate muscle fiber orientation and determine resultant force vectors of muscular components in the quadriceps. ${ }^{33}$ Previous studies have indicated that following muscular injury, fractional anisotropy (FA) values decreased, resulting from increased isotropic diffusion in the region (the result of torn muscle fibers and reduced restricted diffusion of water), and the apparent diffusion coefficient (ADC) values increased, due also to reduced restricted diffusion within that area. ${ }^{34-36}$ One 
specific study evaluated the DTI parameters of muscle tears in the lower limbs of football players and compared them with healthy contralateral muscles. Torn muscle had significantly lower FA and elevated radial diffusivity compared to healthy controls. ${ }^{37}$ These results further validate the feasibility of using DTI to quantify changes in muscle. Zaraiskaya et $\mathrm{al}^{38}$ also imaged injuries of the vastus lateralis muscles, and demonstrated clear differences in FA and ADC values compared to controls. This finding was reinforced by Sinha et $a l,{ }^{39}$ who evaluated the same parameters in the calf muscles and concluded that the microstructural parameters had the ability to track fibers. Therefore, DTI provides a unique opportunity to noninvasively study muscle structure in vivo. Even though it provides unique insight into muscle structure, we were not able to locate any studies that specifically examined the MTrP with DTI.

The studies that examine the structure of muscular injury are of interest because the fibers of injured muscles are more complex and scattered, similar to MTrPs, than those of healthy muscle. Muscle deformation during contraction is characterized by strain tensors. DTI has been used to assess the strain along human muscle, suggesting that this type of analysis could also be a powerful tool when quantifying muscle deformation. ${ }^{40,41}$ Since trigger points have been shown to be stiff taut bands of muscle, DTI could be extremely beneficial as it could characterize the extent of strain within the trigger points, exploring more of their structure. The pennation angle of rat gastrocnemius muscles has also been assessed by DTI and compared with B-mode ultrasound images. This study demonstrated that there was no significant difference between the pennation angle measurements acquired using ultrasound or with DTI. ${ }^{42}$ Studies were conducted by Charles et $\mathrm{al}^{43}$ and Heemskerk et $\mathrm{al}^{44}$ that compared fiber lengths of lower-limb muscles from the DTI images to the lengths of the same muscles from cadaveric (human and mouse, respectively) dissections. On average, the fiber lengths matched well between the two methods. Schenk et al validated DTI fascicle lengths and median pennation angles of the soleus, comparing these to a manual digitization system. ${ }^{45}$ DTI has previously been evaluated in the human forearm and compared with the muscle architecture of a cadaver. In this situation, a combination of T1weighted and diffusion tensor data was able to visualize the architecture and show excellent agreement with cadaver muscles. ${ }^{46}$ Thus, it can be concluded that, DTI compared with cadaveric dissections, show no differences in mean fascicle lengths, but some differences were found for the median pennation angles. The culmination of all these studies is further evidence that DTI-based fiber tracking is a valid and reliable tool for in vivo analysis of pennation angle and fiber length of muscle fibers.

\section{Restrictions and Limitations with DTI}

Although DTI appears to be a reliable method for the study of muscle fiber structure, there are still limitations. One of the difficulties of using DTI is the determination of optimal scan parameters. ${ }^{47}$ Previous studies have indicated an optimal range for $b$ values in terms of minimizing variance $\left(435-725 \mathrm{~s} / \mathrm{mm}^{2}\right),{ }^{48-50}$ and the optimal number of diffusion encoding directions (25). ${ }^{48,49}$ We suggest to use these ranges as a starting point for parameter tuning when imaging muscle. DTI images are typically acquired with a lower resolution than other conventional MRI scans. This can lead to partial volume effects when different fiber orientations are present within a voxel. In particular, regions with crossing fibers may cause an inaccurate representation of fiber directions. ${ }^{51}$ Larger pennation angles have also shown to moderately affect the resolution of the skeletal muscle tractography. ${ }^{52}$

Zeng et $\mathrm{al}^{53}$ identified the crossing fiber limitation when studying rabbit thigh muscle trauma. Significant differences were present between the diffusion parameters of normal and injured muscle; however, there was a drawback within the DTI fiber tracking image. Some of the fibers vanished and/or were weaker in areas. This could be due to a number of factors including $\Delta \mathrm{B}_{\mathrm{o}}$ and $\mathrm{B}_{1}+$ inhomogeneities. But for reliable reconstruction of the smallest fascicles, a higher order model is needed. ${ }^{54}$ Current modelling of DTI can only uncover one direction per voxel, ${ }^{31}$ whereas higher order models provide more gradient directions and can uncover more than one fiber direction per voxel.

\section{HARDI Imaging}

To overcome the limitations of DTI, an alternative method, called high angular resolution diffusion imaging (HARDI), could be used. HARDI is a type of imaging that requires a much larger number of diffusion encoding directions, compared to routine DTI. Our systematic literature search found only one study that used HARDI imaging with human skeletal muscle. Ye et $\mathrm{al}^{54}$ studied the tongue in an attempt to resolve the crossing fiber issue of DTI images by hypothesizing that the HARDI imaging technique could overcome this. However, because of the 
involuntary nature of swallowing, the tongue muscle was not able to remain immobile for the time needed to capture the number of diffusion encoding directions necessary for this technique. Instead, they used a multi-tensor model with a fixed tensor basis that incorporated directional knowledge from the anatomy of the tongue. Their results showed a large increase in the appearance of the crossing fibers. ${ }^{55}$ This suggests that HARDI imaging could be advantageous in the structural evaluation of skeletal muscles since HARDI involves a more complex analytical model and has more encoded directions than routine DTI. During our literature search, we were unable to locate any studies that specifically examined the MTrP using HARDI.

\section{DKI Imaging}

Another potential type of MRI diffusion imaging that could be useful for skeletal muscle microstructure is diffusion kurtosis imaging (DKI). Conventional diffusion MRI assumes that the probability density function of water displacement is Gaussian. DKI shows the heterogeneous diffusion of water more accurately than other techniques. From our literature review, only one study was found that used DKI to assess skeletal muscle. Whole-body maps of the diffusion coefficient, the ADC coefficient and diffusion kurtosis were generated. Goodness of fit was calculated using the sum of squared residuals and was compared between DKI and diffusion weighted imaging. DKI, performed on the erector spinae muscle, demonstrated a strong decrease in the residuals of the fit when compared with diffusion weighted imaging techniques. This indicates that there is a significant contribution of nonGaussian diffusion in muscle tissue, ${ }^{56}$ and could be another advantageous method when evaluating damaged muscle as well.

Other more complex diffusion encoding schemes also exist such as Q-ball imaging and diffusion spectral imaging (DSI). Q-Ball Imaging resolves multiple intravoxel fiber orientations while not assuming any particular diffusivity shape. It requires multiple directions and multiple diffusion shells with some diffusion encoding b-values being $>4000 \mathrm{~s} / \mathrm{mm}^{2}{ }^{57,58}$ DSI studies are two to three times longer than Q-ball, requiring over 200 gradient directions to be sampled using very high diffusion encoding values $\left(8000-12,000 \mathrm{~s} / \mathrm{mm}^{2}\right)$ not typical for routine clinical scanners. Results from DSI are diffusion probability density function (PDF) maps, allowing thorough high- resolution visualization and measurement of fibers and their crossings. 59

\section{Quantitative Review}

We intended to evaluate the benefits of DTI quantitatively, since this seemed to be the most feasible modality when imaging muscular injuries or myopathies. However, due to the heterogeneity of the studies, the grouping of quantitative results for a specific muscle in humans was not attainable. There were many studies assessing how DTI performs within skeletal muscle, but few were performed within the same muscle, in the same species. Within the extracted studies, there were only two studies that had explicitly communicated their healthy and injured quantitative DTI results in the same muscle. This was not enough to perform a detailed, statistically valid quantitative systematic review and meta-analysis.

\section{Discussion}

From the articles reviewed, no articles were found that specifically imaged the microenvironment and structure of the trigger point. Although we cannot make direct inferences on the best technique to image these trigger points, we can make a theoretical suggestion on which technique(s) could provide the most useful information about them. Many of the studies addressed the structure of muscle injury, compared with healthy muscle. We also considered the advantages or disadvantages of modalities. From this, an informed decision on the best technique was made. Studies performed on injured muscle were of particular interest, as the microstructure of injured muscular tissue is disorganized and complex, much like trigger points themselves. Numerous studies, mentioned above, assessed this environment using T2 mapping and DTI, while few have used HARDI or DKI imaging. T2 mapping has permitted quantitation of the extent of muscular injury, but tends to fall short when the goal is to understand the structural nature of the injury. This type of imaging could be useful for imaging edema within trigger points, but in order to fully understand their structural environment, another method needs investigated. DTI has been used to further understand the microstructure of a muscle by allowing researchers to quantify strain parameters and by accurately measuring muscle fibers. As mentioned, these strain parameters are a powerful tool to quantify the extent of deformation during contraction within a muscle. Since trigger points are stiff, contracted areas of muscle, this could be translated to the subject of trigger points, to gain more structural understanding. When 
selecting a modality to image these trigger points, it is essential that the image correctly presents the anatomy of the region and its muscle fibers. Published DTI studies have demonstrated accurate fiber angles and lengths, when relating the images back to cadaveric dissections. Although HARDI and DKI imaging show potential benefits over the other conventional methods, these have not been extensively used in human muscle. Specifically, only one study was found that images human muscle with HARDI, so there are few conclusions about the applicability to image trigger points. HARDI and DKI images also tend to have longer scan times than other MR imaging techniques, which leads to poor clinical utility and may not be feasible for trigger point analysis for patients.

The utilization of advanced MRI techniques such as HARDI or DKI may provide advancements in our understanding of the structure of the myofascial trigger point. At some stage in the future this may have impact on clinical practice with regard to detection of the myofascial trigger point reliably and perhaps personalized treatment formulations based on results of imaging combined with clinical attributes. More basic science research is required prior to achieving any significant clinical impact.

\section{Conclusions}

In conclusion, the review of the current literature on imaging modalities, demonstrates that studies exploring skeletal microstructure and injury using MRI as a diagnostic/ research tool are still scarce. To the best of our knowledge, imaging trigger points to explore their structure using MRI techniques has yet to be explored. Determining the best techniques that image skeletal microstructure can infer which technique would be the most effective at imaging the complex structure of the trigger point. From the review, it is concluded that there has been extensive theoretical and experimental evidence on the benefits of using DTI to explore the length, pennation angle, strain and diffusive properties of fibers. Other techniques such as HARDI and DKI show potential theoretical benefits over DTI but have not been widely used on skeletal microstructure of muscle. These techniques also face a clinical disadvantage because of the longer scan times. Such evidence suggests that further research and studies are necessary to understand the technical optimization of these techniques, and their application in research and eventual clinical practice.

\section{Funding}

No funding was received for the preparation of the manuscript.

\section{Disclosure}

Dr Michael D Noseworthy is the co-founder and CEO of TBIfinder Inc., a Data Analytics Company. None of the authors have any conflict of interest to disclose.

\section{References}

1. Gregory NS, Sluka KA. Anatomical and physiological factors contributing to chronic muscle pain. In: Behavioral Neurobiology of Chronic Pain. Berlin, Heidelberg: Springer; 2014:P327-P348.

2. Sikdar S, Shah JP, Gebreab T, et al. Novel applications of ultrasound technology to visualize and characterize myofascial trigger points and surrounding soft tissue. Arch Phys Med Rehabil. 2009;90 (11):1829-1838. doi:10.1016/j.apmr.2009.04.015

3. Travell JG, Simons DG. Myofascial Pain and Dysfunction: The Trigger Point Manual. Vol. 2. Lippincott Williams \& Wilkins; 1983.

4. Simons DG, Travell JG, Simons LS. Travell \& Simons' Myofascial Pain and Dysfunction: The Trigger Point Manual. 2nd edition. Vol. 1. Baltimore: Williams \& Wilkins; 1999.

5. Kader DF, Wardlaw D, Smith FW. Correlation between the MRI changes in the lumbar multifidus muscles and leg pain. Clin Radiol. 2000;55(2):145-149. doi:10.1053/crad.1999.0340

6. Perez-Palomares S, Oliván-Blázquez B, Arnal-Burró AM, et al. Contributions of myofascial pain in diagnosis and treatment of shoulder pain. A randomized control trial. BMC Musculoskelet Disord. 2009;10(1):92. doi:10.1186/1471-2474-10-92

7. Chen CK, Nizar AJ. Myofascial pain syndrome in chronic back pain patients. Korean $J$ Pain. 2011;24(2):100. doi:10.3344/ kjp.2011.24.2.100

8. Rivers WE, Garrigues D, Graciosa J, Harden RN. Signs and symptoms of myofascial pain: an international survey of pain management providers and proposed preliminary set of diagnostic criteria. Pain Med. 2015;16(9):1794-1805. doi:10.1111/pme.12780

9. Kumbhare DA, Elzibak AH, Noseworthy MD. Assessment of myofascial trigger points using ultrasound. Am J Phys Med Rehabil. 2016;95:72-80. doi:10.1097/PHM.0000000000000376

10. Crema MD, Yamada AF, Guermazi A, Roemer FW, Skaf AY. Imaging techniques for muscle injury in sports medicine and clinical relevance. Curr Rev Musculoskelet Med. 2015;8(2):154-161. doi:10.1007/s12178-015-9260-4

11. Myburgh C, Larsen AH, Hartvigsen J. A systematic, critical review of manual palpation for identifying myofascial trigger points: evidence and clinical significance. Arch Phys Med Rehabil. 2008;89 (6):1169-1176. doi:10.1016/j.apmr.2007.12.033

12. Cojocaru MC, Cojocaru IM, Voiculescu VM, Cojan-Carlea NA, Dumitru VL, Berteanu M. Trigger points - ultrasound and thermal findings. $J$ Med Life. 2015;8(3):315-318.

13. Gerwin RD, Cagnie B, Petrovic M, Van Dorpe J, Calders P, De Meulemeester K. Foci of segmentally contracted sarcomeres in trapezius muscle biopsy specimens in myalgic and nonmyalgic human subjects: preliminary results. Pain Med. 2020;21(10):2348-2356. doi:10.1093/pm/pnaa019

14. Moo EK, Herzog W. Single sarcomere contraction dynamics in a whole muscle. Sci Rep. 2018;8(1):1. doi:10.1038/s41598-018-33658-7

15. Jafari M, Bahrpeyma F, Mokhtari-Dizaji M, Nasiri A. Novel method to measure active myofascial trigger point stiffness using ultrasound imaging. J Bodyw Mov Ther. 2018;22(2):374-378. doi:10.1016/j. jbmt.2017.06.019 
16. Gerwin RD, Dommerholt J, Shah JP. An expansion of Simons' integrated hypothesis of trigger point formation. Curr Pain Headache Rep. 2004;8(6):468-475. doi:10.1007/s11916-004-0069-x

17. Hayashi K, Ozaki N, Kawakita K, et al. Involvement of NGF in the rat model of persistent muscle pain associated with taut band. J Pain. 2011;12(10):1059-1068. doi:10.1016/j.jpain.2011.04.010

18. Higgins JP, Thomas J, Chandler J, et al. Cochrane Handbook for Systematic Reviews of Interventions. Chichester, West Sussex; Hoboken NJ: John Wiley \& Sons; 2008.

19. Downs SH, Black N. The feasibility of creating a checklist for the assessment of the methodological quality both of randomised and non-randomised studies of health care interventions. J Epidemiol Community Health. 1998;52(6):377-384. doi:10.1136/jech.52.6.377

20. Yamada AF, Godoy IR, Neto LP, et al. Diagnostic imaging of muscle injuries in sports medicine: new concepts and radiological approach. Curr Radiol Rep. 2017;5(7):27. doi:10.1007/s40134-017-0223-y

21. McCully KE, Shellock FG, Bank WJ, Posner JD. The use of nuclear magnetic resonance to evaluate muscle injury. Med Sci Sports Exerc. 1992;24(5):537-542. doi:10.1249/00005768-199205000-00007

22. Zhang LY, Ding JT, Wang Y, Zhang WG, Deng XJ, Chen JH. MRI quantitative study and pathologic analysis of crush injury in rabbit hind limb muscles. J Surg Res. 2011;167:e357-63. doi:10.1016/j. jss.2010.09.014

23. Dong YA, Zhang J, Liang W, et al. Magnetic resonance imaging and histopathological analysis of experimental muscle injuries in a rabbit. Biomed Environ Sci. 2013;26(10):841-848. doi:10.3967/bes2013.007

24. Stekelenburg A, Oomens CW, Strijkers GJ, Nicolay K, Bader DL. Compression-induced deep tissue injury examined with magnetic resonance imaging and histology. J App Physiol. 2006;100 (6):1946-1954. doi:10.1152/japplphysiol.00889.2005

25. Gejo R, Kawaguchi Y, Kondoh T, et al. Magnetic resonance imaging and histologic evidence of postoperative back muscle injury in rats. Spine. 2000;25(8):941-946. doi:10.1097/00007632-20000415000008

26. Clarkson PM, Hubal MJ. Exercise-induced muscle damage in humans. Am J Phys Med Rehabil. 2002;81(Suppl):S52-S69. doi:10.1097/00002060-200211001-00007

27. Marqueste T, Giannesini B, Le Fur Y, Cozzone PJ, Bendahan D. Comparative MRI analysis of T2 changes associated with single and repeated bouts of downhill running leading to eccentric-induced muscle damage. J App Physiol. 2008;105(1):299-307. doi:10.1152/ japplphysiol.00738.2007

28. Damon BM, Buck AK, Ding Z. Diffusion-tensor MRI based skeletal muscle fiber tracking. Imag Med. 2011;3(6):675.-687. doi:10.2217/ iim. 11.60

29. Cotten A, Haddad F, Hayek G, Lefebvre G, Dodré E, Budzik JF. Tractography: possible applications in musculoskeletal radiology. Semin Musculoskelet Radiol. 2015;19(4):387-395. doi:10.1055/ s-0035-1563736

30. Galban CJ, Maderwald S, Uffmann K, de Greiff A, Ladd ME. Diffusive sensitivity to muscle architecture: a magnetic resonance diffusion tensor imaging study of the human calf. Eur $J \mathrm{App}$ Physiol. 2004;93(3):253-262. doi:10.1007/s00421-004-1186-2

31. Budzik JF, Balbi V, Verclytte S, Pansini V, Thuc VL, Cotten A. Diffusion tensor imaging in musculoskeletal disorders. Radiograph. 2014;34(3):E56-72. doi:10.1148/rg.343125062

32. Kalia V, Leung DG, Sneag DB, et al. Advanced MRI Techniques for muscle imaging. Semin Musculoskelet Radiol. 2017;21(4):459-469. doi:10.1055/s-0037-1604007

33. Kan JH, Heemskerk AM, Ding Z, et al. DTI-based muscle fiber tracking of the quadriceps mechanism in lateral patellar dislocation. JMRI. 2009;29(3):663-670. doi:10.1002/jmri.21687

34. Cermak NM, Noseworthy MD, Bourgeois JM, et al. Diffusion tensor MRI to assess skeletal muscle disruption following eccentric exercise. Muscle Nerve. 2012;46(1):42-50. doi:10.1002/mus.23276
35. Hata J, Mizuno S, Haga Y, et al. Semiquantitative evaluation of muscle repair by diffusion tensor imaging in mice. JBMR Plus. 2018;2(4):227-234. doi:10.1002/jbm4.10040

36. Heemskerk AM, Damon BM. Diffusion tensor MRI assessment of skeletal muscle architecture. Curr Med Imag Rev. 2007;3 (3):152-160. doi:10.2174/157340507781386988

37. Giraudo C, Motyka S, Weber M, et al. Normalized STEAM-based diffusion tensor imaging provides a robust assessment of muscle tears in football players: preliminary results of a new approach to evaluate muscle injuries. Eur Radiol. 2018;28(7):2882-2889. doi:10.1007/ s00330-017-5218-9

38. Zaraiskaya T, Kumbhare D, Noseworthy MD. Diffusion tensor imaging in evaluation of human skeletal muscle injury. JMRI. 2006;24 (2):402-408. doi:10.1002/jmri.20651

39. Sinha S, Sinha U, Edgerton VR. In vivo diffusion tensor imaging of the human calf muscle. JMRI. 2006;24(1):182-190. doi:10.1002/ jmri.20593

40. Pamuk U, Karakuzu A, Ozturk C, Acar B, Yucesoy CA. Combined magnetic resonance and diffusion tensor imaging analyses provide a powerful tool for in vivo assessment of deformation along human muscle fibers. J Mech Behav Biomed Mat. 2016;63:207-219. doi:10.1016/j.jmbbm.2016.06.031

41. Karakuzu A, Pamuk U, Ozturk C, Acar B, Yucesoy CA. Magnetic resonance and diffusion tensor imaging analyses indicate heterogeneous strains along human medial gastrocnemius fascicles caused by submaximal plantar-flexion activity. J Biomech. 2017;57:69-78. doi:10.1016/j.jbiomech.2017.03.028

42. Damon BM, Ding Z, Anderson AW, Freyer AS, Gore JC. Validation of diffusion tensor MRI-based muscle fiber tracking. MRI Med. 2002;48(1):97-104.

43. Charles JP, Moon CH, Anderst W. Determining subject-specific lower-limb muscle architecture data for musculoskeletal models using diffusion tensor MRI. J Biomech Eng. 2018. doi:10.1115/ 1.4040946

44. Heemskerk AM, Strijkers GJ, Vilanova A, Drost MR, Nicolay K. Determination of mouse skeletal muscle architecture using three-dimensional diffusion tensor imaging. MRI Med. 2005;53(6):1333-1340.

45. Schenk P, Siebert T, Hiepe P, et al. Determination of three-dimensional muscle architectures: validation of the DTI-based fiber tractography method by manual digitization. J Anat. 2013;223(1):61-68. doi:10.1111/joa.12062

46. Froeling M, Nederveen AJ, Heijtel DF, et al. Diffusion-tensor MRI reveals the complex muscle architecture of the human forearm. JMRI. 2012;36(1):237-248. doi:10.1002/jmri.23608

47. Khalil C, Budzik JF, Kermarrec E, Balbi V, Le thuc V, Cotten A. Tractography of peripheral nerves and skeletal muscles. Eur J Radiol. 2010;76(3):391-397. doi:10.1016/j.ejrad.2010.03.012

48. Longwei X. Clinical application of diffusion tensor magnetic resonance imaging in skeletal muscle. Muscles Ligaments Tendons J. 2012;2(1):19.

49. Froeling M, Nederveen AJ, Nicolay K, Strijkers GJ. DTI of human skeletal muscle: the effects of diffusion encoding parameters, signalto-noise ratio and T2 on tensor indices and fiber tracts. NMR Biomed. 2013;26(11):1339-1352. doi:10.1002/nbm.2959

50. Saupe N, Lawrence W, Sussman M, Kassner A, Tomlinson G, Noseworthy M. Diffusion tensor magnetic resonance imaging of the human calf: comparison between $1.5 \mathrm{~T}$ and 3.0T- Preliminary results. Invest Radiol. 2008;43(9):612-618. doi:10.1097/RLI.0b013e31817e909f

51. Bammer R, Acar B, Moseley ME. In vivo MR tractography using diffusion imaging. Eur J Radiol. 2003;45(3):223-234. doi:10.1016/ S0720-048X(02)00311-X

52. Okamoto Y, Okamoto T, Yuka K, Hirano Y, Isobe T, Minami M. Correlation between pennation angle and image quality of skeletal muscle fibre tractography using deterministic diffusion tensor imaging. J Med Imag Rad Oncol. 2012;56(6):622-627. doi:10.1111/ j.1754-9485.2012.02450.x 
53. Zeng H, Zheng JH, Zhang JE, et al. Grading of rabbit skeletal muscle trauma by diffusion tensor imaging and tractography on magnetic resonance imaging. Chinese Medical Sciences Journal= Chung-Kuo I Hsueh K'o Hsueh Tsa Chih. 2006;21(4):276-280.

54. Sieben JM, Van Otten I, Lataster A, et al. In vivo reconstruction of lumbar erector spinae architecture using diffusion tensor MRI. Clin Spine Surg. 2016;29(3):E139-45. doi:10.1097/BSD.00000 00000000036

55. Ye C, Murano E, Stone M, Prince JL. A Bayesian approach to distinguishing interdigitated tongue muscles from limited diffusion magnetic resonance imaging. Comput Med Imag Grap. 2015;45:63-74. doi:10.1016/j.compmedimag.2015.07.005

56. Filli L, Wurnig M, Nanz D, Luechinger R, Kenkel D, Boss A. Whole-body diffusion kurtosis imaging: initial experience on non-Gaussian diffusion in various organs. Invest Radiol. 2014;49 (12):773-778. doi:10.1097/RLI.0000000000000082
57. Barnett A. Theory of Q-ball imaging redux: implications for fiber tracking. Magn Resonan Med. 2009;62(4):910-923. doi:10.1002/ mrm.22073

58. Kuo LW, Chen JH, Wedeen VJ, Tseng WY. Optimization of diffusion spectrum imaging and q-ball imaging on clinical MRI system. Neuroimage. 2008;41(1):7-18. doi:10.1016/j.neuroimage.2008.02.016

59. Wedeen VJ, Wang RP, Schmahmann JD, et al. Diffusion spectrum magnetic resonance imaging (DSI) tractography of crossing fibers. Neuroimage. 2008;41(4):1267-1277. doi:10.1016/j.neuroimage.2008.03.036

60. Liberati A, Altman D, Tetzlaff J, et al. The PRISMA statement for reporting systematic reviews and meta-analyses of studies that evaluate health care interventions: explanation and elaboration. Journal of Clinical Epidemiology. 2009;62(10).

\section{Publish your work in this journal}

The Journal of Pain Research is an international, peer reviewed, open access, online journal that welcomes laboratory and clinical findings in the fields of pain research and the prevention and management of pain. Original research, reviews, symposium reports, hypothesis formation and commentaries are all considered for publication. The manuscript management system is completely online and includes a very quick and fair peer-review system, which is all easy to use. Visit http:// www.dovepress.com/testimonials.php to read real quotes from published authors. 